\title{
LEE011 and ruxolitinib: a synergistic drug combination for natural killer/T-cell lymphoma (NKTCL)
}

\author{
Yan Ting Hee ${ }^{1, *}$, Junli Yan ${ }^{2, *}$, Dean Nizetic ${ }^{1,3}$ and Wee-Joo Chng ${ }^{2,4,5}$ \\ ${ }^{1}$ Lee Kong Chian School of Medicine, Nanyang Technological University, Singapore \\ ${ }^{2}$ Cancer Science Institute of Singapore, National University of Singapore, Singapore \\ ${ }^{3}$ The Blizard Institute, Barts and The London School of Medicine, Queen Mary University of London, London, UK \\ ${ }^{4}$ Department of Haematology-Oncology, National University Cancer Institute of Singapore, National University Health \\ System, Singapore \\ ${ }^{5}$ Department of Medicine, Yong Loo Lin School of Medicine, National University of Singapore, Singapore \\ *These authors have contributed equally to this work \\ Correspondence to: Wee-Joo Chng, email: mdccwj@nus.edu.sg
}

Keywords: NKTCL; LEEO 1 1; ruxolitinib; drug combination; lymphoma

Received: December 28, $2017 \quad$ Accepted: July 12, $2018 \quad$ Published: August 07, 2018

Copyright: Hee et al. This is an open-access article distributed under the terms of the Creative Commons Attribution License 3.0 (CC BY 3.0), which permits unrestricted use, distribution, and reproduction in any medium, provided the original author and source are credited.

\section{ABSTRACT}

Natural killer/T-cell lymphoma (NKTCL) is an aggressive non-Hodgkin lymphoma that has been facing limited success with conventional treatments, urging for the discovery of alternative strategies. Recent studies including ours have revealed that EZH2 and JAK-STAT signalling pathways are key contributors to NKTCL pathogenesis. In particular, we found that EZH2 is overexpressed and directly transcriptionally activates the CCND1 gene to confer growth advantage. CCND1 codes for cyclin D1, which complexes with CDK4/6 to promote $\mathrm{G1}$ to $S$ phase transition. Therefore in this study we investigated whether inhibiting both JAK1/2 and CDK4/6, using LEE011 and ruxolitinib respectively is effective in NKTL. We first demonstrate that separate LEE011 and ruxolitinib treatment is sufficient to cause growth inhibition of NKTCL cells. More importantly, we found that there is synergistic growth inhibitory effects on NKTCL cells with combination treatment of LEE011 and ruxolitinib. The results obtained shows that the targeting of both CDK4/6 and JAK1/2 are promising to develop better treatment alternatives for NKTCL.

\section{INTRODUCTION}

Natural killer/T-cell lymphoma (NKTCL) is an aggressive type of non-Hodgkin lymphoma characterised by a clonal proliferation of NK or T-cells, and patients often have poor survival rates $[1,2]$. To date, treatments for NKTCL are still completely reliant on radiotherapy, chemotherapy or a combination of both, but these have been met with limited success with low complete response rates $[1,3,4]$. There is thus a compelling need to develop alternative strategies for NKTCL.

Recent studies have reported enriched and activated (phosphorylated) STAT3 protein in NKTCL [5-8]. The
JAK-STAT pathway is essential for blood cells to respond to extracellular cytokines via cytokine receptors for proliferation and growth [9]. Signals from these receptors are often propagated through JAKs that can ultimately lead to the increase in cancer cells' survival and proliferative indices via a change in transcription profile through STATs $[10,11]$. The activated STAT3 status was subsequently found to be due to STAT3 activating mutations and/or the loss of receptor-type tyrosine-protein phosphatase $\kappa[7,12$, 13] and could be brought down to normal phosphorylated STAT3 levels upon JAK2 inhibition [7, 13]. These findings thus suggest that the STAT3 pathway could be a promising target for alternative NKTCL treatment. 
Additionally, our previous studies show that the epigenetic writer EZH2 is overexpressed in NKTCL [6, 14]. EZH2 functions in the Polycomb repressive complex 2 (PRC2) along with EED, SUZ12 and RbAp46/48 to trimethylate histone H3 lysine 27 (H3K27me3), a mark often associated with gene repression [15-17]. Contrary to its epigenetic repression function, we have found that $\mathrm{EZH} 2$ directly transcriptionally activates the CCND1 gene independent of its methyltransferase activity in NKTCL [14]. The CCND1 gene codes for cyclin D1, which when complexed with CDK4/6, promotes cell cycle progression from $\mathrm{G} 1$ to $\mathrm{S}$ phase. The overexpression of EZH2 thus likely conferred growth advantage to NKTCL cells by a corresponding upregulation of cyclin D1. Indeed, high levels of CCND1 transcript has been reported in NKTCL cell lines and upregulated cyclin D1 protein levels has been linked to poor prognosis and decreased survival in NKTCL patients $[5,18]$. Hence, targeting CDK4/6 that is downstream of EZH2 could be promising for the treatment of NKTCL by inhibiting cell cycle progression.

Besides being essential to the pathogenesis of NKTCL, the JAK-STAT and EZH2-CDK4/6 pathways were noted to be upstream and downstream in the signalling pathway for cell growth respectively. As such, inhibiting them simultaneously should bring about a more robust and enhanced growth inhibition effect. Here, ruxolitinib and LEE011 (ribociclib) which targets JAK1/2 and CDK4/6 [19, 20] respectively were tested against
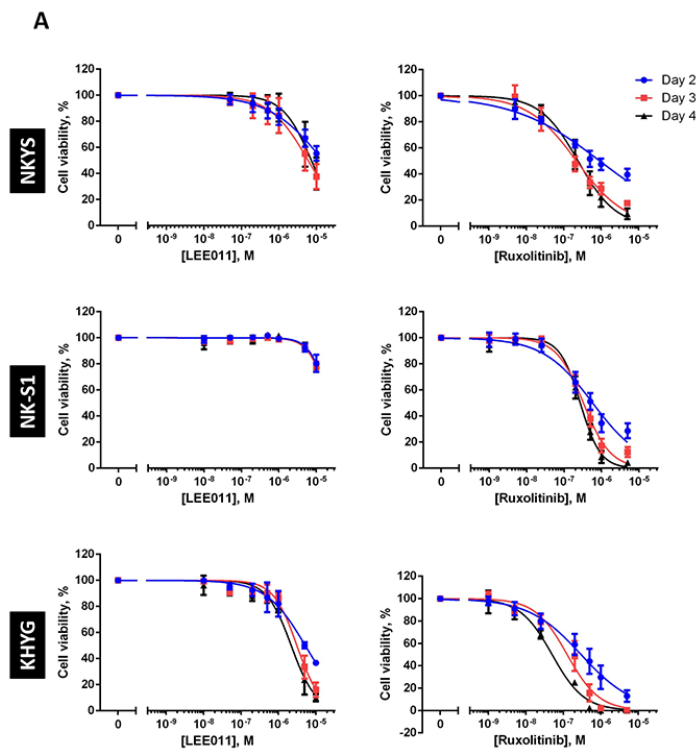

several NKTCL cell lines. Since these two drugs have successfully reached clinical trials, it is hoped that they will show promising results in NKTCL as well. By cell viability assay, it was clearly shown that though these two drugs are able to work individually to inhibit growth of NKTCL cells, a far greater growth inhibition could be achieved when they are used in combination. Changes in apoptotic and proliferative markers and cell cycle analysis further support this observation. These findings thus strongly provide a basis for a promising alternative treatment for NKTCL patients.

\section{RESULTS}

\section{Growth inhibition on NKTCL cell lines was achieved with independent CDK4/6 and JAK1/2 inhibition}

To evaluate growth inhibitory efficacy of CDK4/6 and JAK1/2 inhibition separately, the NKTCL cell lines were treated to a range of LEE011 and ruxolitinib concentrations. Cells were treated over a period of four days, with re-treatment on the second day to account for loss of drug stability over extended time in the incubator. Cell viability was assessed on Day 2, 3 and 4 of treatment. Growth inhibition was achieved with the two drugs independently across almost all the NKTCL cell lines tested as seen in the drop in cell viability as a function

\section{B}
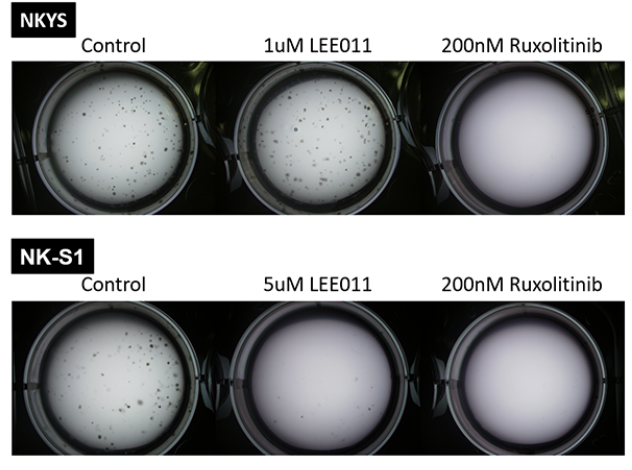

KHYG

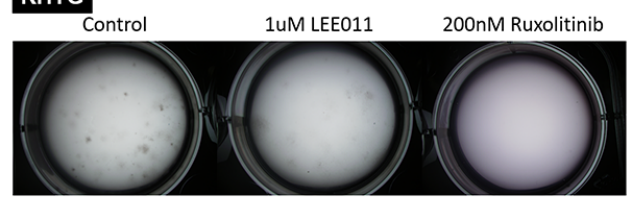

Figure 1: LEE011 and ruxolitinib inhibits growth in NKTCL cell lines. (A) Cell viability assay showed growth inhibition followed after independent LEE011 and ruxolitinib treatment. NKTCL cell lines were separately treated with LEE011 and ruxolitinib and cell viabilities were assessed at Day 2, 3 and 4. In each experiment, triplicate values were averaged and treated wells were normalised against control wells. Data is expressed as mean \pm SEM from three independent experiments. The IC50 curves were plotted based on the variable-slope (four-parameter logistic model), fitted using the least squares model. (B) Clonogenic assay of single LEE011- and ruxolitinib-induced growth inhibition. Cell lines were treated with LEE011 or ruxolitinib at concentrations roughly corresponding to the respective IC50 values determined and checked for colony formation after two weeks $\left(\mathrm{n}=3,{ }^{*} \mathrm{p}<0.05,{ }^{* *} \mathrm{p}<0.01,{ }^{* * *} \mathrm{p}<0.001\right.$, one-way ANOVA test). 
Table 1: IC50 values of LEE011 and ruxolitinib in all NKTCL cell lines tested

\begin{tabular}{|c|c|c|c|c|c|c|}
\hline \multirow{2}{*}{ Day } & \multicolumn{2}{|c|}{ NKYS } & \multicolumn{2}{|c|}{ NK-S1 } & \multicolumn{2}{|c|}{ KHYG } \\
\hline & LEE011/ $\mu \mathrm{M}$ & 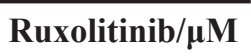 & LEE011/ $\mu \mathrm{M}$ & Ruxolitinib/ $\mu \mathrm{M}$ & LEE011/ $\mu \mathrm{M}$ & Ruxolitinib/ $\mu \mathrm{M}$ \\
\hline 2 & 15.0 & 0.920 & 23.7 & 0.581 & 5.21 & 0.298 \\
\hline 3 & 6.00 & 0.219 & 20.8 & 0.327 & 3.11 & 0.126 \\
\hline 4 & 7.32 & 0.241 & 19.4 & 0.269 & 2.19 & 0.0501 \\
\hline
\end{tabular}

These values were obtained from the plots in Figure 1.

of drug concentration (Figure 1A). From the IC50 curves obtained, the IC50 values of LEE011 and ruxolitinib were then determined for each of the NKTCL cell lines (Table 1).

While the IC50 values of LEE011 fell within the 300 $\mathrm{nM}$ to $3.2 \mathrm{uM}$ that has been determined to be sensitive in neuroblastoma and T-ALL cell lines respectively [21, 22], the concentrations were nonetheless in the micromolar range. To confirm if the decrease in cell viability resulted from LEE011 is due to inhibition of its intended targets, we thus performed knockdown (KD) with CDK4/6 siRNA which was compared to negative control siRNA. Decrease in cell viability followed after CDK4/6 KD (Supplementary Figure 1A). We also noted the extent of the drop in cell viability seemed to correlate with CDK4/6 $\mathrm{KD}$ efficiency (Supplementary Figure 1B) as the less efficient CDK6 KD in KHYG is reflected in the least drop in cell viability. This thus suggests that the effect of LEE011 is likely due to CDK4/6 inhibition.

Growth inhibitory efficacies of the two agents were also then demonstrated in clonogenic assays which served to detect cells that have survived and retained progenyproducing capacity following treatment [23]. Each cell line was treated with LEE011 and ruxolitinib separately at roughly the IC50 values obtained (Table 1) and was left to grow in the incubator for two weeks. In accordance to the cell viability assays, lesser number of colonies were observed in treated wells as compared to control wells across most NKTCL cell lines tested (Figure 1B). These results thus demonstrated that LEE011 and ruxolitinib are able to inhibit NKTCL cell growth individually.

\section{LEE011 and ruxolitinib-induced growth inhibition is mediated through blocking cell cycle progression and STAT signalling respectively}

Next, we studied how growth inhibition is achieved by LEE011 and ruxolitinib independently in NKTCL. For LEE011, it likely prevents progression into S phase by targeting CDK $4 / 6$ and inhibiting complex formation with cyclin D1 [19]. Indeed, upon LEE011 treatment, decreased cyclin E2 and phosphorylated retinoblastoma $(\mathrm{Rb})$ levels were noted (Figure 2A). The mechanism of action by ruxolitinib, however, is not as clear due to JAK1/2 involvement in other pathways besides STAT signalling.
In particular, JAK2 phosphorylation of EZH2 at tyrosine 641 is able to promote $\mathrm{EZH} 2$ degradation via the $\mathrm{SCF}^{\beta}{ }^{-\mathrm{TrCP}}$ E3 ubiquitin ligase pathway in B cell lymphoma [24]. In addition, JAK3 phosphorylation of EZH2 was found to mediate EZH2 switch to non-canonical function [25]. It would thus be interesting to see if similar JAK2-mediated EZH2 degradation exists in NKTCL or that EZH2 switch to non-canonical function can be mediated by JAK $1 / 2$ as well. Gaining insights into the ruxolitinib effects on EZH2 activity is essential given EZH2's key role in NKTCL oncogenesis [14].

It was first confirmed if EZH2 was able to transcriptionally activate $C C N D 1$ gene expression. For this, control plasmids or plasmids containing EZH2 wild-type (WT) sequence were transfected into NKYS, followed by measuring changes in CCND1 mRNA levels (Figure 2B). As seen, overexpression of EZH2 WT led to a corresponding increase in CCND1 mRNA levels, affirming EZH2's non-canonical function as transcriptional activator. This result provided proof that the non-canonical function of EZH2 can be monitored by cyclin D1 protein levels. Next, the three NKTCL cell lines were subjected to single ruxolitinib treatment at a concentration close to the IC50 values and changes in protein levels were studied. If a JAK2-mediated EZH2 degradation exists, an increase in the levels of EZH2, cyclin D1 and the H3K27me3 mark that EZH2 catalyses would be expected upon JAK2 inhibition by ruxolitinib. Likewise, if JAK1/2 phosphorylation is able to cause a functional switch in EZH2 activity, an increase in $\mathrm{H} 3 \mathrm{~K} 27 \mathrm{me} 3$ levels should follow after ruxolitinib treatment as EZH2 would be redirected back to catalyse the deposition of the H3K27me3 mark. None of these trends were seen in all the NKTCL cell lines tested (Figure 2C), indicating that JAK1/2 regulation on EZH2's activity is absent in NKTCL. It is affirmative that a successful JAK1/2 inhibition by ruxolitinib was achieved as seen by the decrease in p-STAT3/5 protein levels between control and treated cells. To note, KHYG was revealed to have a far lower STAT3/5 basal phosphorylation level than the other two cell lines (Supplementary Figure 2), which might account for the lower ruxolitinib IC50 concentration. Nonetheless, LEE011 and ruxolitinib worked as intended to cause growth inhibition in NKTCL. 


\section{Combination treatment of LEE011 and ruxolitinib induced synergistic growth inhibitory effects}

Using the IC50 values of the two drugs determined for each NKTCL cell line, a range of concentrations around the IC50 value was selected for combination treatment. In contrast to the treatment conditions for single treatments, cells were only treated for a maximum period of three days, as any enhanced inhibitory effects should be observed at an earlier time point if LEE011 and ruxolitinib work synergistically with each other. As before, cells were re-treated at Day 2 and cell viabilities were assessed at Day 2 and 3 (Figure 3A). As seen, at a certain concentration of LEE011, a further drop in cell viability was noted when the cells were treated with ruxolitinib as well, and vice versa. These results thus suggest that combining LEE011 and ruxolitinib is able to produce an enhanced growth inhibitory effect in NKTCL cell lines.

However, simply by just noting the further drop in cell viability in combined as compared to single treatment is not sufficient to conclude on the synergism with using LEE011 and ruxolitinib together. To this end, determination of combination indices (CIs) of the two drugs at all combination concentrations was performed. A CI value of more than, equal and smaller than 1 is indicative of antagonism, additive and synergism respectively [26]. As most of the CI values obtained are less than 1, it strongly suggests that there is synergism at most concentrations of the two drugs and the two time points at which cell viabilities were assessed (Figure
3B, refer to Supplementary Table 1 for the numerical CI values).

After noting synergistic growth inhibitory effect of LEE011 and ruxolitinib via cell viability assay, it is then imperative to understand how enhanced growth inhibition is achieved. For this purpose, we studied changes in the expression levels of proliferative and apoptotic markers in the NKTCL cell lines following drug treatment for three days (Figure 3C). Changes in cells' proliferative capacity were assessed by the levels of the proliferative marker Ki-67 and cell-death inhibitor survivin [27, 28], while cleaved PARP and caspase 9 were probed for any increase in apoptosis $[29,30]$. In general, we noticed a decrease in $\mathrm{Ki}-67$ and survivin levels from control to treated cells as early as $10 \mathrm{~h}$ following treatment. Importantly, Ki67 and survivin fell more in combination treatments compared to that of single treatments from around $10 \mathrm{~h}$ that persisted up until $72 \mathrm{~h}$, suggesting that the synergistic effect of LEE011 and ruxolitinib occurs at an early time point. As for the apoptotic markers, slightly higher levels of cleaved caspase 9 for all treated cells in contrast to control arose at $24 \mathrm{~h}$. From $48 \mathrm{~h}$, higher levels of cleaved caspase 9 were detected in cells treated with both drugs than cells that received single drug. Cleaved PARP levels remained relatively constant throughout all time points in NKYS and NK-S1 cells, but increased further in combination treatment from 48 $\mathrm{h}$ in KHYG cells. These results thus provide promising leads into the mechanism of growth inhibition of LEE011 and ruxolitinib combination treatment.

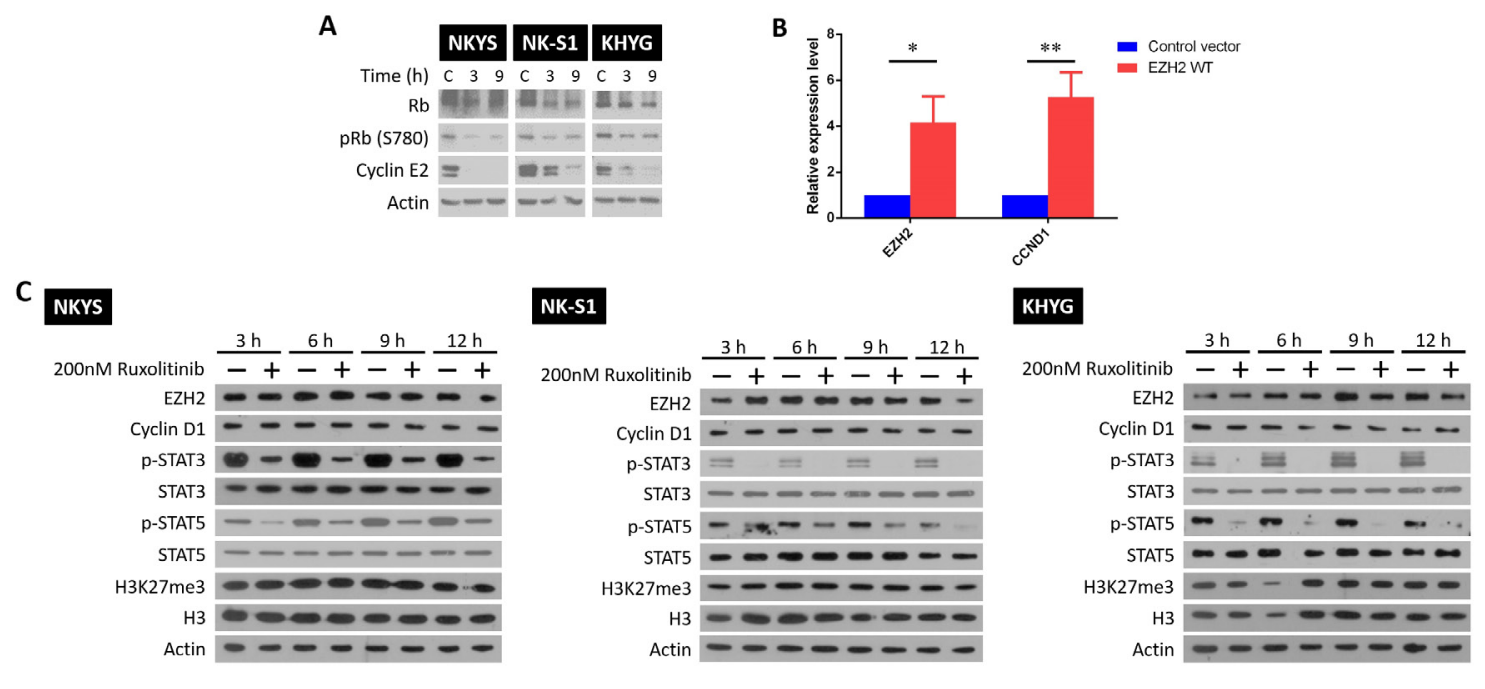

Figure 2: LEE011 and ruxolitinib inhibit growth by blocking cell cycle progression and STAT signalling respectively. (A) Western blot showing that LEE011 blocks CDK4/6 phosphorylation of Rb and the subsequent expression of cyclin E2. Cells were treated with their respective LEE011 IC50 concentrations and harvested 3 and $9 \mathrm{~h}$ after. (B) RT-qPCR results of mRNA harvested from NKYS cells that were either transfected with control and EZH2 WT plasmids after $24 \mathrm{~h}$. Relative fold change of EZH2 and CCND1 mRNA levels were obtained by normalising to control cells. Differences were determined to be statistically significant $\left(\mathrm{n}=3,{ }^{*} \mathrm{p}<0.05,{ }^{* *} \mathrm{p}<0.01\right.$, one-tailed student's $t$-test). (C) NKYS, NK-S1 and KHYG cells were treated with $200 \mathrm{nM}$ ruxolitinib and harvested after 3, 6, 9 and $12 \mathrm{~h}$ later. Blots shown for each cell line are representative blots from the same experiment, among two other independent biological replicates. 
Beside studying levels of proliferative and apoptotic markers, knowing at which stage of the cell cycle is growth inhibited is also important for us to further understand the mechanism of action of LEE011 and ruxolitinib in NKTCL. Thus, we carried out flow cytometric analysis of cell cycle populations on KHYG after treatment for $48 \mathrm{~h}$ (Figure 3D). As seen, there was an increase in sub-G1 population and decrease in both $\mathrm{S}$ and $\mathrm{G} 2 / \mathrm{M}$ phase populations going from single to combination treatment. Particularly the decrease in $\mathrm{S}$ phase between single LEE011 and combination treatment was significant. In summary, this finding further corroborated the synergistic relationship between LEE011 and ruxolitinib, in that inhibiting JAK $1 / 2$ on top
A
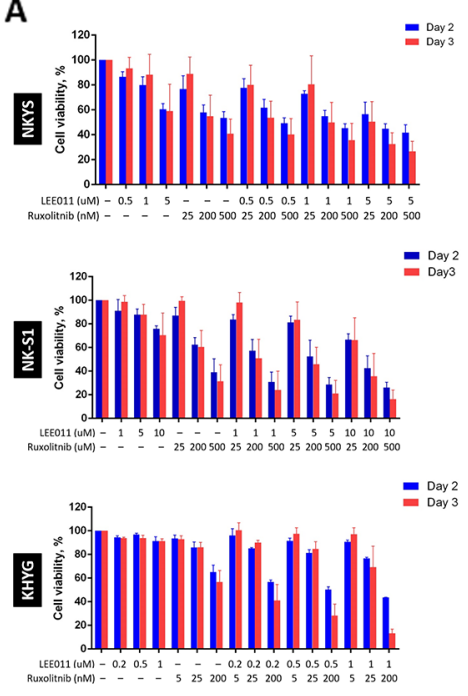

Day 2
B
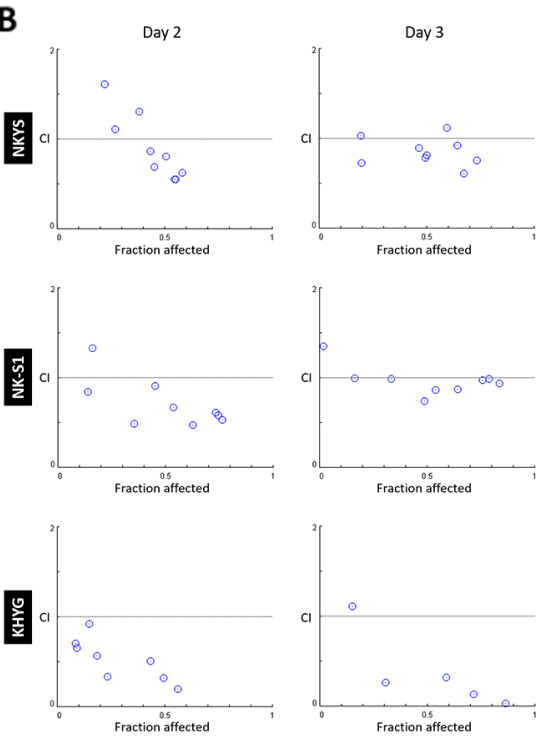

D

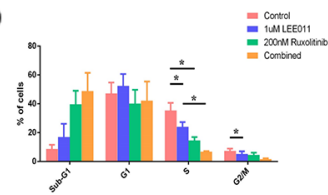

C

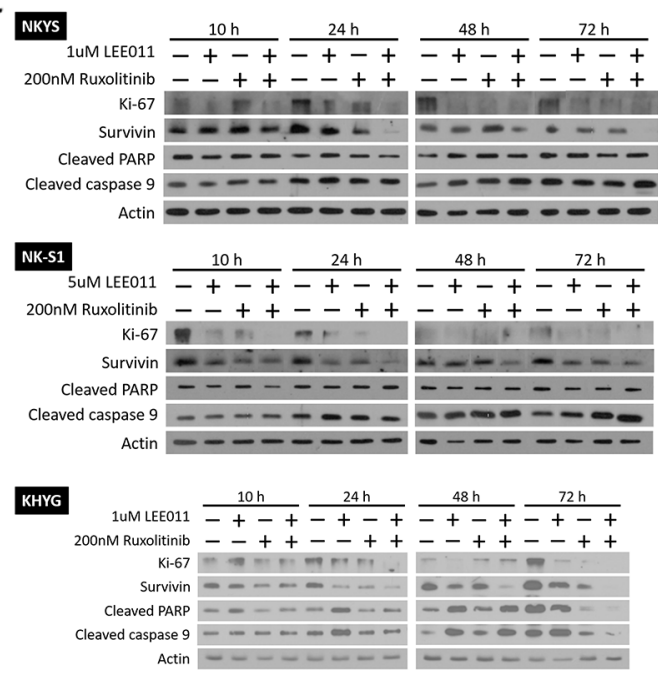

Figure 3: LEE011 and ruxolitinib displayed synergistic relationship on achieving growth inhibition in NKTCL cell lines. (A) Cell viability assay showed enhanced growth inhibition with dual LEE011 and ruxolitinib treatment. The cells were subjected to single or combined LEE011 treatment as indicated and cell viabilities were assessed on Day 2 and 3. In each experiment, values from triplicate wells were averaged and treatment wells were normalised against control wells. Data is expressed as mean \pm SEM from three independent experiments. (B) Fa-CI plots for the cells on the days cell viabilities were assessed revealed a synergistic growth inhibition effect with dual treatment of LEE011 and ruxolitinib. In the Fa-CI plots, the dashed line where CI $=1$ indicates an additive reaction between the two drugs. Points that fall above $(\mathrm{CI}>1)$ and below $(\mathrm{CI}<1)$ the dashed line denote antagonism and synergism respectively. $(\mathbf{C})$ Western blot analysis of proliferative and apoptotic markers following LEE011 and ruxolitinib combined treatment. Cells were singly and combine treated to LEE011 and ruxolitinib at concentrations as indicated. Protein lysates from 10 and $24 \mathrm{~h}$ were ran on the same SDS-PAGE gel, while that of 48 and $72 \mathrm{~h}$ were ran on a separate gel. In general, a decrease in proliferative markers and increase in apoptotic markers in combine treatment as compared to single treatment were noted across different time points. (D) Evaluation of combination treatment using flow cytometric analysis of cell cycle populations. KHYG cells were treated with LEE011 and ruxolitinib at concentrations as indicated. Significant decrease in S phase populations was noted between single LEE011 and combine treatment $\left(n=3,{ }^{*} \mathrm{p}<0.05\right.$, one-way ANOVA test). 
of CDK4/6 could further prevent entry into S phase of the cell cycle.

\section{DISCUSSION}

The low success rates that current treatments on NKTCL are showing $[1,3,4]$ has urged us to discover better treatment alternatives. Several key findings in recent years have helped to shed light on the pathogenesis of NKTCL. In particular, deregulation of JAK-STAT signalling as well as overexpression of cyclin D1 by upregulated EZH2 levels have been noted to confer oncogenic potential in NKTCL $[7,14]$. These findings thus suggested for the dual targeting of JAKs and cyclin D1 in NKTCL.

Here, we tested a combination of ruxolitinib and LEE011 that inhibit JAK1/2 and CDK4/6 respectively against several NKTCL cell lines, namely NKYS, KHYG and NK-S1. We first confirmed the growth inhibitory efficacy of separate LEE011 and ruxolitinib treatment via cell viability assays and clonogenic assay. The higher IC50 concentrations noted for LEE011 as compared to ruxolitinib across all NKTCL cell lines tested might suggest a possibility that inhibition of JAK $1 / 2$ produces a more potent effect than CDK4/6 inhibition. This might be explained by the coupling of JAKs to the activation of pathways that promote cell survival [10]. Therefore, while LEE011 and ruxolitinib both decrease cell proliferation, ruxolitinib can affect survivability as well.

The effect that LEE011 has on NK-S1 was observably less effective than in NKYS and KHYG, with just about $20 \%$ decrease in cell viability at concentrations as high as $10 \mu \mathrm{M}$, which is also reflected in a higher IC50 value. This difference in LEE011 growth inhibitory efficacy between the NKTCL cell lines could be due to the mutational status of JAK3 in them. While NKYS and KHYG possess wild-type JAK3, NK-S1 carries a homozygous $J A K 3^{A 572 V}$ mutation, which makes JAK3 constitutively active [31]. The constitutively active JAK3 could allow for the persistence of pro-survival and proproliferation signals in NK-S1 cells, attenuating the effect of LEE011.

Subsequently, it was concluded that a synergistic relationship exists between LEE011 and ruxolitinib as the CI values were less than 1 at most concentrations tested. Still the presence of CI values greater than 1 should not be ignored. In general, a CI value of more than 1 was observed when low concentrations of LEE011 and ruxolitinib were used. However, this does not mean that the two drugs work antagonistically at low concentrations and synergistically at high concentrations. Rather, it is plausible that the growth inhibitory effects obtained at these low concentrations in combination were not very different from that of single treatment. Any increase in cell viability in combination from single treatment at low concentrations could be the result of experimental variations.

Similarly, the strikingly high CI values in KHYG cells (Supplementary Table 1, highlighted in grey) could be due to the same reasons as well. In particular, the $5 \mathrm{nM}$ ruxolitinib dose might have been too low to be included for the assessment of combination effect. This is in view that the IC50 concentrations determined for ruxolitinib at Day 2 and 3 were close to $200 \mathrm{nM}$, which is 40 -fold higher than $5 \mathrm{nM}$. This could thus account for the high CIs noted at $5 \mathrm{nM}$ ruxolitinib at varying LEE011 concentrations. Considering that $\mathrm{CI}$ values were less than 1 in most other instances where sufficiently high concentrations of LEE011 and ruxolitinib were used, it is thus sound to conclude that there is a synergistic effect of inhibiting both CDK4/6 and JAK1/2 in NKTCL.

The synergistic relationship between LEE011 and ruxolitinib was further confirmed by cell cycle analysis, with significant difference going from single to combination treatment noted at $\mathrm{S}$ phase. Synergism was also evidenced by changes in proliferation and apoptotic markers, which showed that combination treatment causes further decrease in proliferation and increase in apoptosis. Difference in Ki-67 levels arose as early as $10 \mathrm{~h}$, suggesting that the synergistic effect of LEE011 and ruxolitinib in inhibition of proliferation occurs at an early time point. This was then followed by a loss of the anti-apoptotic molecule survivin at $24 \mathrm{~h}$. The loss of survivin then possibly promoted apoptotic death as seen by an increase in cleaved caspase 9 levels from $48 \mathrm{~h}$ onwards. Cell death appears to have occurred via the intrinsic apoptotic pathway, given caspase 9's exclusive involvement in only this apoptotic pathway [29]. Therefore, the examination of the proliferative and apoptotic markers also revealed the synergistic relationship between LEE011 and ruxolitinib in NKTCL.

The results of LEE011 and ruxolitinib combination treatment thus provided insights into a promising new treatment for NKTCL. The effect of JAK $1 / 2$ inhibition with ruxolitinib agrees with other studies where NKTCL growth inhibition was similarly achieved via JAK2 inhibition using AG490 [7, 8]. However, ruxolitinib is favoured here as AG490 is less potent and has been reported to block many other Ser/Thr kinases, which would complicate results interpretation [32]. Our data on ruxolitinib in NKTCL are also in concordance with recent publications $[33,34]$. In addition, the combination use of LEE011 and ruxolitinib have been demonstrated to be successful in the treatment of myelofibrosis and is currently undergoing phase I of clinical trials $[35,36]$. It is thus hoped that the success of LEE011 and ruxolitinib combination use for myelofibrosis can be leveraged upon to push forward an alternative treatment for NKTCL.

Single ruxolitinib treatment caused no change in $\mathrm{EZH} 2$, cyclin D1 and $\mathrm{H} 3 \mathrm{~K} 27 \mathrm{me} 3$ levels, indicating absence of JAK1/2 regulation on EZH2's level and 
activity. However, it is possible that JAK3 is the predominent regulator of EZH2 activity in NKTCL and hence no changes were observed following JAK $1 / 2$ inhibition. This is consistent with our previous observation that inhibition of JAK3 with JAK3 inhibitor PF956980 can target deregulated EZH2 pathway using [25].

\section{MATERIALS AND METHODS}

\section{Cell culture}

The cell lines used in this study included NKYS, NK-S1 [37, 38] (both NK lymphoma cell lines and KHYG [39] (NK leukaemia cell line). NKYS and KHYG cells were cultured in Roswell Park Memorial Institution media (RPMI; Hyclone, USA) supplemented with 10\% heatinactivated fetal bovine serum (FBS; Hyclone), 1\% each of penicillin-streptomycin $(\mathrm{P} / \mathrm{S})$ and L-glutamine (both from Biowest, France). NKYS and KHYG were also supplemented with $20 \mathrm{ng} / \mathrm{ml}$ and $40 \mathrm{ng} / \mathrm{ml}$ recombinant human interleukin-2 (IL-2; Miltenyi Biotec, Germany) respectively. NK-S1 was cultured in Dulbecco's Modified Eagles Medium (DMEM; Hyclone) without sodium pyruvate, supplemented with 10\% FBS (Hyclone), 10\% inactivated horse serum (Life Technologies, USA) and 1\% each of $\mathrm{P} / \mathrm{S}$ and L-glutamine (Biowest). All cell lines were grown in humidified incubators at $37^{\circ} \mathrm{C}$ with $5 \% \mathrm{CO}_{2}$.

\section{Drugs}

Drugs used in this study included the CDK4/6 inhibitor, LEE011 (Novartis, Switzerland), and the JAK1/2 inhibitor, ruxolitinib (Invivogen, USA).

\section{Cell viability assay}

Cells were seeded at $2 \times 10^{4}$ cells $/ 100 \mu \mathrm{l} /$ well in 96-well plates in triplicates and treated with or without the respective drugs at various concentrations for a maximum period of four days. The cells were re-treated at Day 2 to account for loss of drug stability in the incubator over extended time period. Cell viability was assessed at Day 2, 3 and 4 using the CellTiter-Glo 2.0 assay (Promega, USA) as per manufacturer's protocol and luminescence was read using the Infinite M200 plate reader (Tecan, Switzerland).

\section{Protein concentration estimation}

Cells were lysed in radioimmunoprecipitation assay buffer and were subjected to sonication. Protein lysates were 10-fold diluted and standards were prepared by serial dilution of $1 \mathrm{mg} / \mathrm{ml}$ bovine serum albumin (BSA; Hyclone). Standards and samples were all done in triplicates. $200 \mu \mathrm{l}$ of Quick Start ${ }^{\text {TM }}$ Bradford reagent (Bio-Rad, USA) was then added and the intensity of the resulting blue colour was measured at a wavelength of $595 \mathrm{~nm}$.

\section{Western blotting}

Appropriate percentage of sodium dodecyl sulfatepolyacrylamide gel electrophoresis (SDS-PAGE) was used to resolve equal amounts of protein samples. The resolved protein samples were then transferred to polyvinylidene difluoride membrane (Bio-Rad). After which, the membrane was blocked in 5\% BSA solution and incubated overnight with primary antibodies at $4^{\circ} \mathrm{C}$, followed by incubation for 1 hour with secondary antibodies at room temperature. Primary antibodies used included BioLegend antibody Ki67 (652402), Cell Signalling antibody cyclin D1 (2978), cleaved caspase 9 (9501), cleaved poly(ADP-ribose) polymerase (PARP; 9541), EZH2 (3147), H3 (9715), H3K27me3 (9733, 9756), p-STAT3 (9131, sc-8059), p-STAT5 (9351, 9356), survivin (2802), and SantaCruz antibody actin (sc-1615HRP), CDK4 (sc-23896), CDK6 (sc-7961), GAPDH (sc-47724 HRP), STAT3 (sc-482), STAT5 (sc-835). Secondary antibodies used included SantaCruz antibody goat anti-rabbit IgG-HRP (sc-2030) and goat anti-mouse IgG-HRP (sc-2031). Blotted proteins were detected and visualised using Amersham ${ }^{\text {TM }}$ ECL ${ }^{\text {TM }}$ start Western Blotting detection reagent (GE Healthcare, UK), Luminata ${ }^{\mathrm{TM}}$ Crescendo Western HRP substrate (Millipore, USA) or SuperSignal ${ }^{\mathrm{TM}}$ West Femto Maximum Sensitivity Substrate (Thermo Scientific, USA). Where necessary, p-STAT3 and p-STAT5 antibodies were stripped using Restore ${ }^{\mathrm{TM}}$ Western Blot Stripping Buffer (Thermo Scientific) following manufacturer's recommendations to re-probe for STAT3 and STAT5 respectively.

\section{Clonogenic assay}

5000 NKYS, KHYG and NK-S1 cells were seeded separately into each well of a 6-well plate in StemMACS hematopoietic stem cell-colony forming units (HSCCFU) basic methylcellulose medium (Miltenyi Biotec). Appropriate concentration of IL-2, LEE011 and ruxolitinib were also added into each well. The plates were placed in humidified incubators at $37^{\circ} \mathrm{C}$ with $5 \% \mathrm{CO}_{2}$ for two weeks, after which colony formation was scored with an inverted microscope.

\section{Plasmid and siRNA transfection}

Transfection on NKTCL cell lines were done using Neon ${ }^{\circledR}$ transfection system (Invitrogen) following manufacturer's protocol at the electroporation parameters of $1250 \mathrm{~V}, 10 \mathrm{~ms}$ width and three pulses. For each transfection, $1 \times 10^{6}$ cells were used. For overexpression experiment, NKYS cells were transfected with $2 \mu \mathrm{g}$ control (pCMV-HA-GFP) or EZH2 (pCMV-HAEZH2WT; kindly gifted by Dr Yu Qiang of GIS) vector plasmid, $0.5 \mu \mathrm{g}$ green fluorescence protein plasmid (pMAX-GFP) and $0.5 \mu \mathrm{g}$ puromycin-resistant plasmid. For knockdown experiment, $200 \mathrm{nM}$ human non-targeting siRNA negative control \#1 (4390844) or $100 \mathrm{nM}$ each of 
siRNA against CDK4 (s2822) and CDK6 (s51) were used and were all purchased from Life Technology Ambion.

\section{Quantitative RT-PCR}

Cells were lysed using QIAzol lysis reagent (Qiagen, Germany) and total RNA was isolated using the RNeasy Mini Kit (Qiagen). The extracted RNA was then converted to cDNA with the iScript Reverse Transcription Supermix (Bio-Rad). The mRNA levels of EZH2, CCND1 and housekeeping gene GAPDH were then quantitated using iTaq ${ }^{\mathrm{TM}}$ Universal SYBR ${ }^{\circledR}$ Green Supermix (BioRad) according to manufacturer's recommendations, using their respective primers (Supplementary Table 2).

\section{Flow cytometric assay for cell cycle}

To measure the effect of LEE011 and ruxolitinib combinatorial treatment on apoptosis, NKYS, KHYG and NK-S1 were separately treated at $2 \times 10^{5}$ cells $/ \mathrm{ml}$ in $10 \mathrm{~cm}$ dishes at appropriate drug concentrations, and harvested $48 \mathrm{~h}$ later. Cells were washed and fixed in ice-cold $70 \%$ ethanol for $2 \mathrm{~h}$ before further incubation at $-20^{\circ} \mathrm{C}$ for up to $24 \mathrm{~h}$. Immediately before flow cytometric analysis, the cells were treated with RNase A (Qiagen) and stained with propidium iodide (Invitrogen, USA) for $30 \mathrm{~min}$. Collection and analysis of data was done using BD LSR II Special Order System (BD Biosciences, USA).

\section{Statistical analysis}

All graphs are expressed as mean \pm SEM, from three independent experiments. Difference between samples were evaluated by unpaired one-tailed student's $t$-test or one-way ANOVA test where appropriate, and p-values $<0.05$ were considered as statistically significant. IC50 curves (variable slope) were plotted using GraphPad Prism 6.1 (GraphPad Software, USA) and IC50 values over different treatment time points were compared via the extra sum-of-squares F test. Synergistic effects from drug combination was determined via the quantitative method using CompuSyn [26] (version 1.0, ComboSyn, Inc.).

\section{Author contributions}

JY and WJC conceived, designed and supervised the study. YTH performed the experiments, analysed and interpreted the data. YTH, JY and WJC wrote the paper. YTH and JY contributed equally to this work.

\section{ACKNOWLEDGMENTS AND FUNDING}

This study was supported by the National Medical Research Council (NMRC) grants NMRC/Clinician Scientist-Individual Research/1343/2012 (WJC), NMRC/ Basic Research Grant-New Investigator/2021/2014 (JY) and the Singapore Ministry of Education Academic Research Fund Tier 2 grant MOE2015-T2-2-119 (DN). WJC was also supported by the NMRC Clinician Scientist Investigator Award. We thank Savita Nandal at Medical Oncology, Novartis (Singapore) Pte Ltd for kindly providing us LEE011.

\section{CONFLICTS OF INTEREST}

The authors declare no conflicts of interest.

\section{REFERENCES}

1. Chaudhary RK, Bhatt VR, Vose JM. Management of extranodal natural killer/t-cell lymphoma, nasal type. Clin Lymphoma Myeloma Leuk. 2015; 15:245-52.

2. Lee J, Suh C, Park YH, Ko YH, Bang SM, Lee JH, Lee DH, Huh J, Oh SY, Kwon HC, Kim HJ, Lee SI, Kim JH, et al. Extranodal natural killer T-cell lymphoma, nasal-type: a prognostic model from a retrospective multicenter study. $\mathrm{J}$ Clin Oncol. 2006; 24:612-8.

3. Moon JH, Lee BH, Kim JA, Lee YJ, Chae YS, Yhim HY, Kwak JY, Do YR, Park Y, Song MK, Shin HJ, Kim T, Lee $\mathrm{JJ}$, et al. Clinical impact of induction treatment modalities and optimal timing of radiotherapy for the treatment of limited-stage NK/T cell lymphoma. Leuk Res. 2016; 49:80-7.

4. Tse E, Kwong YL. How I treat NK/T-cell lymphomas. Blood. 2013; 121:4997-5005.

5. Huang Y, de Reynies A, de Leval L, Ghazi B, Martin-Garcia N, Travert M, Bosq J, Briere J, Petit B, Thomas E, Coppo P, Marafioti T, Emile JF, et al. Gene expression profiling identifies emerging oncogenic pathways operating in extranodal NK/T-cell lymphoma, nasal type. Blood. 2010; 115:1226-37.

6. Ng SB, Selvarajan V, Huang G, Zhou J, Feldman AL, Law M, Kwong YL, Shimizu N, Kagami Y, Aozasa K, SaltoTellez M, Chng WJ. Activated oncogenic pathways and therapeutic targets in extranodal nasal-type NK/T cell lymphoma revealed by gene expression profiling. J Pathol. 2011; 223:496-510.

7. Coppo P, Gouilleux-Gruart V, Huang Y, Bouhlal H, Bouamar H, Bouchet S, Perrot C, Vieillard V, Dartigues P, Gaulard P, Agbalika F, Douay L, Lassoued K, et al. STAT3 transcription factor is constitutively activated and is oncogenic in nasal-type NK/T-cell lymphoma. Leukemia. 2009; 23:1667-78.

8. Tsutsui M, Yasuda H, Suto H, Imai H, Isobe Y, Sasaki M, Kojima Y, Oshimi K, Sugimoto K. Frequent STAT3 activation is associated with Mcl-1 expression in nasal NK-cell lymphoma. Int J Lab Hematol. 2010; 32:419-26.

9. Robb L. Cytokine receptors and hematopoietic differentiation. Oncogene. 2007; 26:6715-23.

10. Chen E, Staudt LM, Green AR. Janus kinase deregulation in leukemia and lymphoma. Immunity. 2012; 36:529-41. 
11. Darnell JE Jr. STATs and gene regulation. Science. 1997; 277:1630-5.

12. Chen YW, Guo T, Shen L, Wong KY, Tao Q, Choi WW, Au-Yeung RK, Chan YP, Wong ML, Tang JC, Liu WP, Li GD, Shimizu N, et al. Receptor-type tyrosine-protein phosphatase kappa directly targets STAT3 activation for tumor suppression in nasal NK/T-cell lymphoma. Blood. 2015; 125:1589-600.

13. Küçük C, Jiang B, Hu X, Zhang W, Chan JK, Xiao W, Lack N, Alkan C, Williams JC, Avery KN, Kavak P, Scuto A, Sen E, et al. Activating mutations of STAT5B and STAT3 in lymphomas derived from $\gamma \delta$-T or NK cells. Nat Commun. 2015; 6:6025.

14. Yan J, Ng SB, Tay JL, Lin B, Koh TL, Tan J, Selvarajan V, Liu SC, Bi C, Wang S, Choo SN, Shimizu N, Huang G, et al. EZH2 overexpression in natural killer/T-cell lymphoma confers growth advantage independently of histone methyltransferase activity. Blood. 2013; 121:4512-20.

15. Cao R, Wang L, Wang H, Xia L, Erdjument-Bromage H, Tempst P, Jones RS, Zhang Y. Role of histone H3 lysine 27 methylation in Polycomb-group silencing. Science. 2002; 298:1039-43.

16. Kuzmichev A, Nishioka K, Erdjument-Bromage H, Tempst P, Reinberg D. Histone methyltransferase activity associated with a human multiprotein complex containing the Enhancer of Zeste protein. Genes Dev. 2002; 16:2893-905.

17. Muller J, Hart CM, Francis NJ, Vargas ML, Sengupta A, Wild B, Miller EL, O'Connor MB, Kingston RE, Simon JA. Histone methyltransferase activity of a Drosophila Polycomb group repressor complex. Cell. 2002; 111:197-208.

18. Cao W, Liu Y, Zhang H, Wang S, Zhang L, Zhang L, Sun B. Expression of LMP-1 and Cyclin D1 protein is correlated with an unfavorable prognosis in nasal type NK/T cell lymphoma. Mol Med Rep. 2008; 1:363-8.

19. Gelbert LM, Cai S, Lin X, Sanchez-Martinez C, Del Prado M, Lallena MJ, Torres R, Ajamie RT, Wishart GN, Flack RS, Neubauer BL, Young J, Chan EM, et al. Preclinical characterization of the CDK4/6 inhibitor LY2835219: in-vivo cell cycle-dependent/independent anti-tumor activities alone/in combination with gemcitabine. Invest New Drugs. 2014; 32:825-37.

20. Quintas-Cardama A, Vaddi K, Liu P, Manshouri T, Li J, Scherle PA, Caulder E, Wen X, Li Y, Waeltz P, Rupar M, Burn T, Lo Y, et al. Preclinical characterization of the selective JAK1/2 inhibitor INCB018424: therapeutic implications for the treatment of myeloproliferative neoplasms. Blood. 2010; 115:3109-17.

21. Rader J, Russell MR, Hart LS, Nakazawa MS, Belcastro LT, Martinez D, Li Y, Carpenter EL, Attiyeh EF, Diskin SJ, Kim S, Parasuraman S, Caponigro G, et al. Dual CDK4/CDK6 inhibition induces cell-cycle arrest and senescence in neuroblastoma. Clin Cancer Res. 2013; 19:6173-82.
22. Pikman Y, Alexe G, Roti G, Conway AS, Furman A, Lee ES, Place AE, Kim S, Saran C, Modiste R, Weinstock DM, Harris M, Kung AL, et al. Synergistic Drug Combinations with a CDK4/6 Inhibitor in T-cell Acute Lymphoblastic Leukemia. Clin Cancer Res. 2017; 23:1012-1024.

23. Franken NA, Rodermond HM, Stap J, Haveman J, van Bree C. Clonogenic assay of cells in vitro. Nat Protoc. 2006; 1:2315-9.

24. Sahasrabuddhe AA, Chen X, Chung F, Velusamy T, Lim MS, Elenitoba-Johnson KS. Oncogenic Y641 mutations in EZH2 prevent Jak2/beta-TrCP-mediated degradation. Oncogene. 2015; 34:445-54.

25. Yan J, Li B, Lin B, Lee PT, Chung TH, Tan J, Bi C, Lee XT, Selvarajan V, Ng SB, Yang H, Yu Q, Chng WJ. EZH2 phosphorylation by JAK3 mediates a switch to noncanonical function in natural killer/T-cell lymphoma. Blood. 2016; 128:948-58.

26. Chou TC, Talalay P. Quantitative analysis of dose-effect relationships: the combined effects of multiple drugs or enzyme inhibitors. Adv Enzyme Regul. 1984; 22:27-55.

27. Reed JC, Reed SI. Survivin' cell-separation anxiety. Nat Cell Biol. 1999; 1:E199-200.

28. Scholzen T, Gerdes J. The Ki-67 protein: from the known and the unknown. J Cell Physiol. 2000; 182:311-22.

29. Elmore S. Apoptosis: a review of programmed cell death. Toxicol Pathol. 2007; 35:495-516.

30. Oliver FJ, de la Rubia G, Rolli V, Ruiz-Ruiz MC, de Murcia G, Murcia JM. Importance of poly(ADP-ribose) polymerase and its cleavage in apoptosis. Lesson from an uncleavable mutant. J Biol Chem. 1998; 273:33533-9.

31. Koo GC, Tan SY, Tang T, Poon SL, Allen GE, Tan L, Chong SC, Ong WS, Tay K, Tao M, Quek R, Loong S, Yeoh KW, et al. Janus kinase 3-activating mutations identified in natural killer/T-cell lymphoma. Cancer Discov. 2012; 2:591-7.

32. Pattison MJ, Mackenzie KF, Arthur JS. Inhibition of JAKs in macrophages increases lipopolysaccharide-induced cytokine production by blocking IL-10-mediated feedback. J Immunol. 2012; 189:2784-92.

33. Dufva O, Kankainen M, Kelkka T, Sekiguchi N, Awad SA, Eldfors S, Yadav B, Kuusanmaki H, Malani D, Andersson EI, Pietarinen P, Saikko L, Kovanen PE, et al. Aggressive natural killer-cell leukemia mutational landscape and drug profiling highlight JAK-STAT signaling as therapeutic target. Nat Commun. 2018; 9:1567.

34. Kuusanmäki H, Dufva O, Parri E, van Adrichem AJ, Rajala H, Majumder MM, Yadav B, Parsons A, Chan WC, Wennerberg K, Mustjoki S, Heckman CA. Drug sensitivity profiling identifies potential therapies for lymphoproliferative disorders with overactive JAK/STAT3 signaling. Oncotarget. 2017; 8:97516-97527. https://doi. org/10.18632/oncotarget.22178.

35. Bose P, Verstovsek S. Myelofibrosis: an update on drug therapy in 2016. Expert Opin Pharmacother. 2016; 17:2375-2389. 
36. Rampal RK, Maria P, Amritha Varshini HS, Levine RL, Cao A. Synergistic therapeutic efficacy of combined JAK1/2, pan-PIM, and CDK4/6 inhibition in myeloproliferative neoplasms. Blood. 2016; 128:634.

37. Loong SL, Hwang JS, Lim ST, Yap SP, Tao M, Chong TW, Tan LH, Huynh H. An Epstein-Barr virus positive natural killer lymphoma xenograft derived for drug testing. Leuk Lymphoma. 2008; 49:1161-7.

38. Tsuchiyama J, Yoshino T, Mori M, Kondoh E, Oka T, Akagi T, Hiraki A, Nakayama H, Shibuya A, Ma Y, Kawabata T,
Okada S, Harada M. Characterization of a novel human natural killer-cell line (NK-YS) established from natural killer cell lymphoma/leukemia associated with Epstein-Barr virus infection. Blood. 1998; 92:1374-83.

39. Yagita M, Huang CL, Umehara H, Matsuo Y, Tabata R, Miyake M, Konaka Y, Takatsuki K. A novel natural killer cell line (KHYG-1) from a patient with aggressive natural killer cell leukemia carrying a p53 point mutation. Leukemia. 2000; 14:922-30. 